\title{
Activated Integrin-Linked Kinase Negatively Regulates Muscle Cell Enhancement Factor 2C in C2C12 Cells
}

\author{
Zhenguo Dong, Wei Pan, Haiqing Wu, Dongjun Liu, and Ming Cang \\ Key Laboratory of Mammalian Reproductive Biology and Biotechnology, Ministry of Education, Inner Mongolia University, \\ Inner Mongolia, Hohhot 010021, China \\ Correspondence should be addressed to Ming Cang; ndcangming@sina.com
}

Received 3 October 2015; Revised 25 November 2015; Accepted 29 November 2015

Academic Editor: Goutam Ghosh Choudhury

Copyright (c) 2015 Zhenguo Dong et al. This is an open access article distributed under the Creative Commons Attribution License, which permits unrestricted use, distribution, and reproduction in any medium, provided the original work is properly cited.

\begin{abstract}
Our previous study reported that muscle cell enhancement factor 2C (MEF2C) was fully activated after inhibition of the phosphorylation activity of integrin-linked kinase (ILK) in the skeletal muscle cells of goats. It enhanced the binding of promoter or enhancer of transcription factor related to proliferation of muscle cells and then regulated the expression of these genes. In the present investigation, we explored whether ILK activation depended on PI3K to regulate the phosphorylation and transcriptional activity of MEF2C during C2C12 cell proliferation. We inhibited PI3K activity in C2C12 with LY294002 and then found that ILK phosphorylation levels and MEF2C phosphorylation were decreased and that MCK mRNA expression was suppressed significantly. After inhibiting ILK phosphorylation activity with Cpd22 and ILK-shRNA, we found MEF2C phosphorylation activity and MCK mRNA expression were increased extremely significantly. In the presence of Cpd22, PI3K activity inhibition increased MEF2C phosphorylation and MCK mRNA expression indistinctively. We conclude that ILK negatively and independently of PI3K regulated MEF2C phosphorylation activity and MCK mRNA expression in $\mathrm{C} 2 \mathrm{C} 12$ cells. The results provide new ideas for the study of classical signaling pathway of PI3K-ILK-related proteins and transcription factors.
\end{abstract}

\section{Introduction}

PI3K plays a key regulatory role in the proliferation and differentiation of skeletal muscle cells $[1,2]$. Studies have shown that PI3K signaling is crucial for the development of muscle by directly or indirectly influencing phosphorylation of proteins such as PKB and ILK, playing an important role in proliferation, differentiation, and survival of muscle cells [35]. PI3K also interacts with transcription factors or proteins related to proliferation of muscle cells. Tamir and Bengal [6] found that the phosphorylation of the transcription factor MEF2C was suppressed after inhibition of PI3K activity.

Activation of ILK stimulated by growth factor is normally regulated in a PI3K-dependent manner via phosphatidylinositol $(3,4,5)$-trisphosphate (PIP3) interaction with the central pleckstrin homology- (PH-) like domain of ILK $[7,8]$. ILK plays a very important role in myocardial regeneration [9], angiogenesis and invasion and metastasis of tumor cells [10], and matrix adhesion and signal transduction [11, 12]. Activated ILK directly affects protein phosphorylation and regulates cellular functions. ILK regulates the phosphorylation of Akt at Ser473 and of glycogen synthase kinase 3 (GSK3) in various cell types [13]. We found that ILK also directly regulates MEF2C phosphorylation in the skeletal muscle cells of goats [14].

The MEF2 proteins (MEF2A-D) consist of a family of transcriptional factors that play a central role during the development of skeletal muscle [15]. The DNA binding site of MEF2 includes a conserved DNA sequence CTA (A/T) 4TAG, present in the regulatory regions of muscle-specific genes $[16,17]$. Studies have shown that the phosphorylation of the serine residue (S59) between MADS and MEF2 domain in MEF2C would enhance the DNA binding activity of $\mathrm{MEF} 2 \mathrm{C}$, and the residue in the MEF2 family members is conservative [18]. MEF2C interacts with the A/T enrichment region in the enhancer of the skeletal muscle-specific creatine kinase enhancer (MCK) and thus regulates and activates the expression of muscle-specific genes [19].

In summary, PI3K plays an important role in the proliferation of skeletal muscle cells. PI3K has a great impact on ILK 
activation. ILK also controls MEF2C-mediated regulation of skeletal muscle cell proliferation, following inhibition of phosphorylation. However, no report is available on PI3Kdependent activation of ILK directly regulating MEF2C phosphorylation during the proliferation of skeletal muscle cells. The present study explored whether activated ILK directly regulated the phosphorylation of MEF2C during the proliferation of $\mathrm{C} 2 \mathrm{C} 12$ cells and regulates the expression of myogenic genes.

\section{Materials and Methods}

2.1. Materials. DMSO (Wako, KPM0962, Japan), ILK antibody (Abcam, ab15838, USA), ILK phosphorylation antibody (sc-130196), and polyclonal antibody p-MEF-2 (Ser59) (sc-13919-R) were purchased from Santa (USA); MEF2C antibody (ab64644) and $\alpha$-tubulin antibody (ab15246) were purchased from Abcam (USA).

2.2. PI3K Inhibitor and ILK Inhibitor. A pharmacological inhibitor specific for the PI3K pathway, namely, 2-(4morpholino)-8-phenyl-4H-1-benzopyran-4-one (LY294002), was used. Applicable cultures were supplemented with LY294002 at indicated concentrations, $30 \mathrm{~min}$ prior to treatment $[20,21]$. A pharmacological inhibitor specific for the ILK, N-methyl-3-(1-(4-(piperazin-1-yl)phenyl)-5-( $4^{\prime}-($ trifluoromethyl)-[1,1'-biphenyl]-4-yl)-1H-pyrazol-3-yl)propanamide (22) (Cpd22, Compound 22), facilitated the dephosphorylation of Akt at Ser473 and other ILK targets [22]. LY294002 was obtained from Santa Cruz Biotechnology and Cpd22 was obtained from Calbiochem, which were prepared and stored according to manufacturer's instructions.

2.3. Cell Culture. $\mathrm{C} 2 \mathrm{C} 12$ cells were planted in $10 \mathrm{~cm}$ culture dish $\left(1 \times 10^{6}\right.$ cells per dish $)$ and maintained at $37^{\circ} \mathrm{C}$ and $5 \% \mathrm{CO}_{2}$ in a humidified atmosphere and cultured in Dulbecco's Modified Eagle's Medium (DMEM) (Invitrogen) supplemented with $12 \%$ fetal bovine serum (Hyclone) and $1 \%$ penicillin/streptomycin (Sigma Aldrich).

2.4. MTT Cell Activity. Subsequent to different treatments, supernatants were discarded and MTT solution (5 mg $\mathrm{MTT} / \mathrm{mL}$ PBS) was added to each well containing the cell monolayer and incubated for different times at $37^{\circ} \mathrm{C}$ in a humidified $5 \% \mathrm{CO}_{2}$ atmosphere. After incubation, formazan crystals generated in healthy cells were dissolved in $0.6 \mathrm{~mL}$ of DMSO and analyzed to determine absorbance at $490 \mathrm{~nm}$ versus blank. Absorbance values were calculated as a percentage versus untreated controls. MTT was obtained from Sigma Aldrich (the result is in Supplementary Material available online at http://dx.doi.org/10.1155/2015/ 748470).

2.5. ILK RNA Interference. Three ILK short hairpin RNA (shRNA) sequences were designed as follows, with nucleotide start positions based on the NCBI entry for ILK (accession NM_001161724.1): ILK-810, 5'-GGCAGGGCAATGATATTGTTG-3'; ILK-999, 5' -GATCTCTCTACAATGTTCTAC-3'; ILK-1048, $5^{\prime}$-GAGCCAAGCTGTAAAGTTTGC$3^{\prime}$; and ILK-1763, 5'-CCCGCCTGTCACAATAAAGTT-3'.
And shRNA negative control (NC-shRNA) is $5^{\prime}$-GTTCTCCGAACGTGTCACGT-3'. The sequences were subcloned into PGPU6/GFP/Neo vectors (GenePharma, China) and transiently transfected into $\mathrm{C} 2 \mathrm{C} 12$ cultures.

2.6. Western Blot. Following treatment, cells were immediately transferred to ice and rinsed in $0.5 \mathrm{~mL}$ of RIPA lysis buffer (Millipore, USA). Lysates were diluted in $5 \times$ SDSPAGE sample loading buffer (Beijing ComWin Biotech Co., Ltd., China) and heated in a boiling water bath for 35 minutes in order to fully denature protein, followed by sodium dodecyl sulfate polyacrylamide gel electrophoresis (SDS-PAGE) using 12\% polyacrylamide gels. Gels were run for $30 \mathrm{~min}$ at $90 \mathrm{~V}$ and $60 \mathrm{~min}$ at $120 \mathrm{~V}$. Following SDSPAGE, proteins were transferred to polyvinylidene fluoride (PVDF) membranes (Immobilon, Millipore, USA) using a semidry electrotransfer system (Bio-Rad, USA) for $60 \mathrm{~min}$ at $0.3 \mathrm{~A}$ (constant current). Membranes were then incubated with specific primary antibodies diluted as 1:500-1:1000 in TBS-T $(1: 1000)$, overnight at $4^{\circ} \mathrm{C}$. The next day, membranes were incubated in anti-rabbit (or anti-goat) horseradish peroxidase-conjugated secondary antibody (Abcam, USA) for $1 \mathrm{~h}$ at room temperature. Antibodies were detected with the Pierce ECL Western Blotting Substrate as per the manufacturer's instructions, and bands were exposed to autoradiography film (Tanon 5200, Yuanpinghao Biotechnology Co., Ltd., China). Exposed bands were visualized and then quantified by densitometry using Image J (National Institutes of Health, USA). All bands were expressed as optical density values relative to a control on the same blot.

2.7. $q P C R$ Analysis. Following treatment, the total RNA of cells was immediately extracted. Reverse transcription using $500 \mathrm{ng}$ total RNA was performed with PrimeScript RT Master Mix (Takara). The MCK (GenBank Acc.: NM_007710.2) primer sequences were designed by GenePharma (China): forward primer (F): 5'-GAGGCAATATGAAGGAGGTTTTCC- $3^{\prime}$; reverse primer $(\mathrm{R}): 5^{\prime}$ GGTGCTCGTTCCACATGAAGG-3'. The GAPDH (GenBank Acc.: NM 001289726.1) primer sequences were designed by Beijing Genomics Institute (BGI) (China): forward primer (F): $5^{\prime}$-TGTGTCCGTCGTGGATCTGA-3' ${ }^{\prime}$; reverse primer (R): $5^{\prime}$-TTGCTGTTGAAGTCGCAGGAG-3'. The qPCR experiments used SYBR Premix Ex Taq (Takara) and the real-time fluorescent quantitative PCR of JENA (qTOWER 2.2, Germany). PCR conditions were $95^{\circ} \mathrm{C}$ for $30 \mathrm{~s}$ for the initial denaturation step, followed by an appropriate number of cycles at $95^{\circ} \mathrm{C}$ for $10 \mathrm{~s}$ and $60^{\circ} \mathrm{C}$ for $30 \mathrm{~s}$. Threshold cycles (Ct) were automatically derived from the amplification plots constructed from the ROX-normalized fluorescence signals by MxPro qPCR software. Relative quantification was performed by the comparative Ct $\left(2^{-\Delta \Delta C t}\right)$ method $(n=3)$. GAPDH was used to normalize the values.

2.8. Statistical Analysis. Data are presented as means \pm SD ( $n=3$ for each group). The significance of differences between experimental or treated groups and control groups 


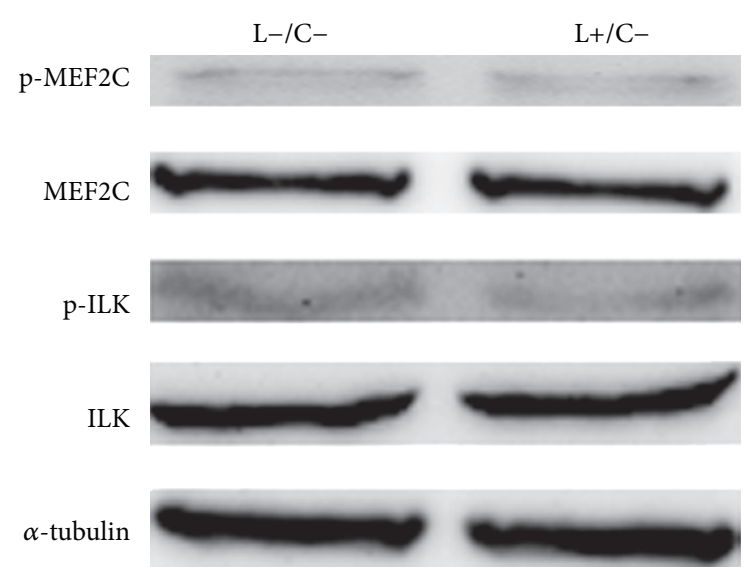

(a)

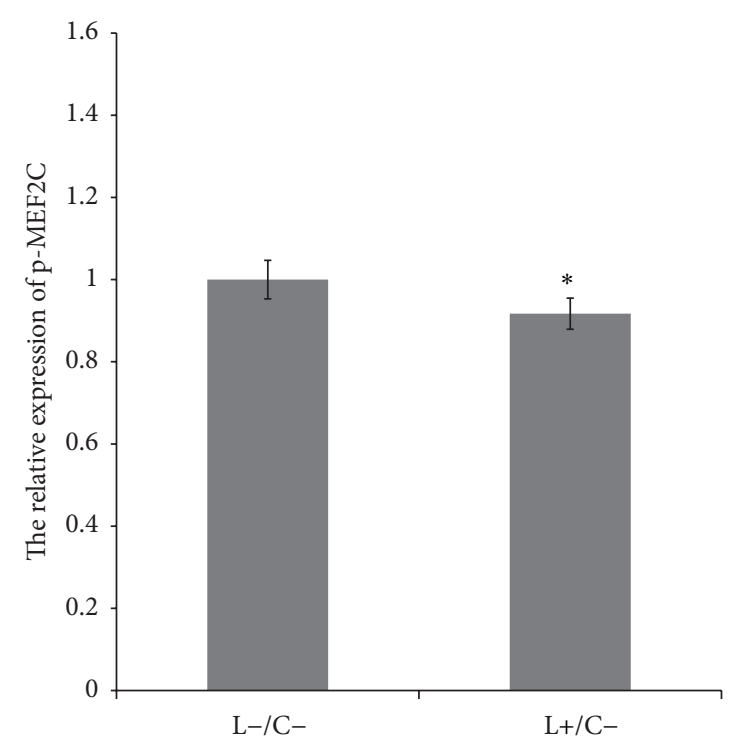

(c)

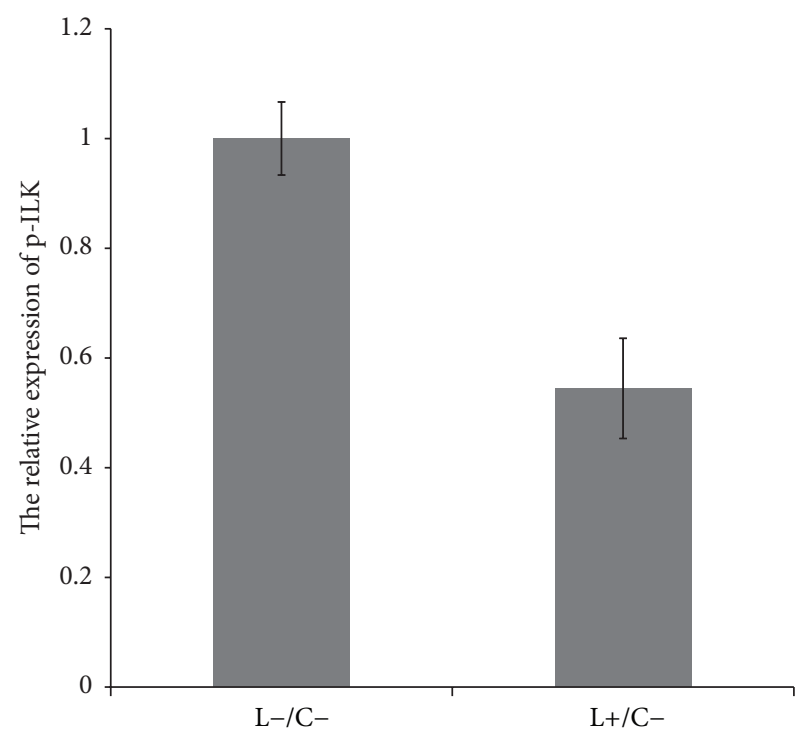

(b)

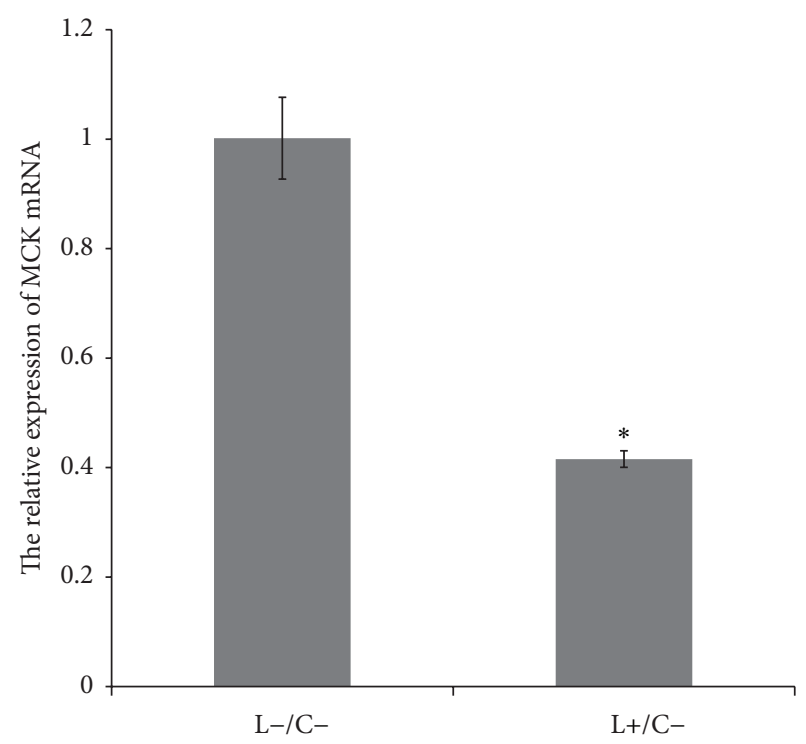

(d)

FIGURE 1: Inhibition of PI3K inhibited ILK and MEF2C phosphorylation activity and the expression of MCK mRNA. (a), (b), and (c) Changes in ILK and MEF2C expression in LY294002-treated (L+/C-) C2C12 cells were not obvious; ILK $(0.544445 \pm 0.091, p>0.05)$ and MEF2C $(0.9170784 \pm 0.038, p<0.05)$ phosphorylation levels decreased. The $3 \mathrm{~h}$ treatment with $20 \mu \mathrm{M}$ LY294002 had a clearly inhibitory effect on ILK phosphorylation and MEF2C phosphorylation levels. (d) MCK mRNA levels in C2C12 cells treated with LY294002 (L+/C-) were significantly lower $(0.4156358 \pm 0.015, p<0.05)$ than that in the control $(\mathrm{L}-/ \mathrm{C}-)$.

was determined by analysis of variance; ${ }^{*} p<0.05,{ }^{* *} p<0.01$ by the $t$-test were considered statistically significant.

\section{Results}

3.1. Inhibition of PI3K-Dependent ILK Phosphorylation Inhibited MEF2C Phosphorylation Activity and the Expression of $M C K m R N A$. We used $20 \mu \mathrm{M}$ LY294002 to treat C2C12 for $3 \mathrm{~h}$ to inhibit the activity of PI3K. As illustrated in Figures 1(a) and 1(b), ILK expression did not change after the inhibition of PI3K activity, but the ILK phosphorylation decreased $(0.544445 \pm 0.091, p>0.05)$.

As demonstrated in Figures 1(a) and 1(c), the expression of MEF2C protein did not change after the inhibition of PI3K activity, whereas the phosphorylation level of MEF2C (S59) levels decreased significantly $(0.9170784 \pm 0.038, p<0.05)$. MEF2C interacted with the enhancer region of MCK and thus enhanced the expression of MCK, and the expression of MCK was decreased significantly $(0.4156358 \pm 0.015$, $p<0.05)$. 


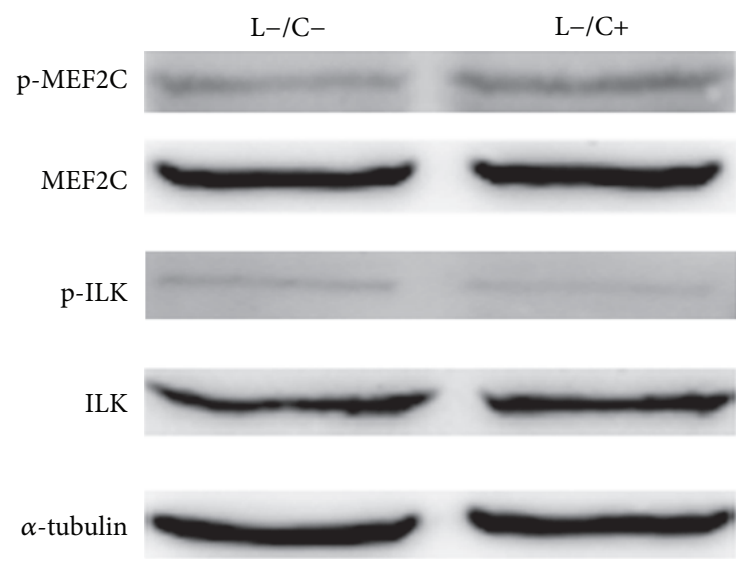

(a)

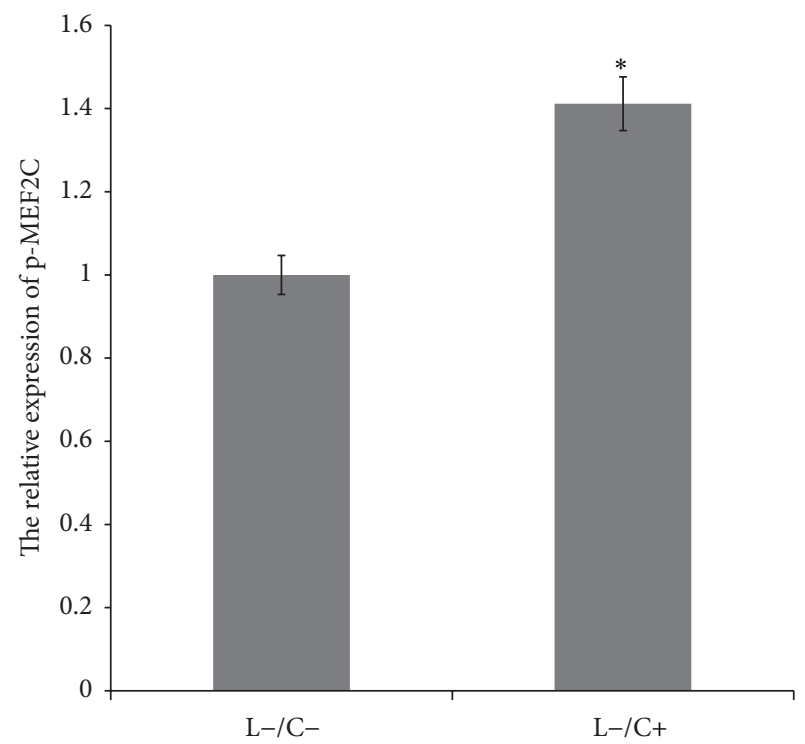

(c)

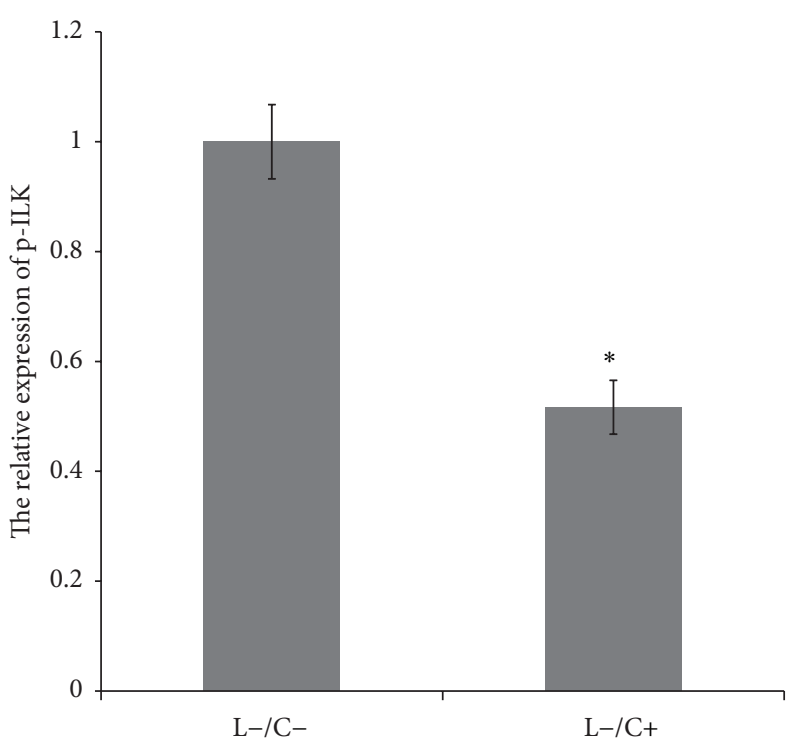

(b)

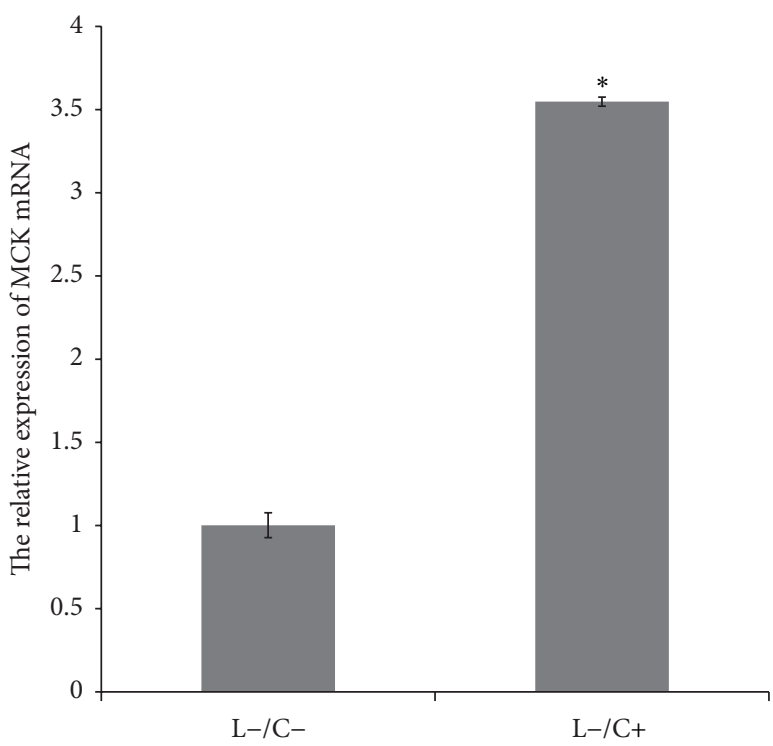

(d)

FIGURE 2: Inhibition of ILK phosphorylation activity increased MEF2C phosphorylation activity and the expression of MCK mRNA. (a), (b), and (c) Changes in ILK and MEF2C expression in Cpd22-treated (L-/C+) C2C12 cells were not obvious; ILK $(0.516547 \pm 0.098, p<0.05)$ phosphorylation levels decreased significantly and MEF2C phosphorylation levels increased significantly $(1.4117083 \pm 0.065, p<0.05)$. (d) MCK mRNA levels in C2C12 cells treated with Cpd22 were significantly increased $(3.5478187 \pm 0.027, p<0.05)$ compared with that in the control.

\subsection{ILK Regulated MEF2C Negatively}

3.2.1. After the Direct Inhibition of ILK Phosphorylation, MFE2C Phosphorylation Activity and the Expression of MCK $m R N A$ Were Significantly Increased. After treatment of C2C12 with $10 \mu \mathrm{M}$ Cpd22, ILK expression did not change (Figures 2(a) and 2(b)), while ILK phosphorylation level significantly decreased $(0.516547 \pm 0.098, p<0.05)$. As shown in Figures 2(a) and 2(c), the expression of MEF2C was not altered after the addition of ILK inhibitor Cpd22, while the phosphorylation level of MEF2C (S59) increased significantly $(1.4117083 \pm 0.065, p<0.01)$ to 1.41 times that of the control group. As shown in Figure 2(d), the expression of MCK was 3.55 times that of the untreated control group, and the expression of MCK was significantly increased (3.5478187 \pm 0.027, $p<0.05)$.

3.2.2. ILK-shRNA Enhanced MEF2C Phosphorylation Activity and MCK mRNA Expression in C2C12. After four ILKshRNA (ILK-810, ILK-999, ILK-1048, and ILK-1763) and NCshRNA were transfected and untransfected control cells (control) were transiently transfected into $\mathrm{C} 2 \mathrm{C} 12$ for $48 \mathrm{~h}$, control $(1.00177 \pm 0.073)$ and NC-shRNA $(1.005488 \pm 0.060)$ had similar levels of ILK mRNA $(p>0.05)$, but four ILK-shRNA 
had different levels of ILK mRNA (Figure 3(a)): ILK-810 $(0.6749447 \pm 0.0916712, p<0.05)$; ILK-999 (0.480587304 \pm $0.015, p<0.05) ;$ ILK-1048 (0.2506583 $\pm 3.50213 E-05$, $p<0.01)$; ILK-1763 (0.2732058 $\pm 0.0006739, p<0.01)$. The similarity of the western blot results (Figure 3(b)) showed that ILK-1048 was the most potent one and was selected for further study.

We transfected C2C12 with ILK-1048 for $48 \mathrm{~h}$, and then there was an obvious decrease in ILK expression and ILK phosphorylation activity $(0.45204 \pm 0.027, p<0.05)$ compared with those of sh-NC (Figures 3(c) and 3(d)). However, MEF2C phosphorylation activity $(1.463772 \pm 0.022, p<0.01)$ was increased distinctly (Figure 3(e)), and MCK mRNA levels $(1.829917 \pm 0.070, p<0.01)$ were significantly higher than that of the control (Figure $3(\mathrm{f})$ ). The similarity of the ILKshRNA results to that of the drug treatments suggested that ILK downregulates MEF2C.

3.3. Inhibition of PI3K-Dependent Activation of ILK Did Not Directly and Negatively Regulate MEF2C Phosphorylation Activity and MCK Expression. Using Cpd22, C2C12 was further treated with LY294002. As illustrated in Figures 4(a) and 4(b), ILK expression did not change, while ILK phosphorylation level was significantly decreased $(0.557457 \pm$ $0.066, p<0.05)$.

As shown in Figures 4(a) and 4(c), the expression of $\mathrm{MEF} 2 \mathrm{C}$ did not change after the addition of 2 inhibitors, whereas the phosphorylation level of MEF2C (S59) increased partly $(1.1359755 \pm 0.054, p>0.05)$. As illustrated in Figure $4(\mathrm{~d})$, the expression of MCK was 1.71 times that of control group after addition of two kinds of inhibitors $(1.7096923 \pm 0.020, p>0.05)$, but it was lower than that of the experimental group following a single treatment with Cpd22 (3.55 times). Moreover, it was significantly lower than that of the experimental group following single treatment using Cpd22, which is consistent with the MEF2C protein analysis.

\section{Discussion}

The $\mathrm{C} 2 \mathrm{C} 12$ cell line is an immortalized rat skeletal muscle satellite cell primarily isolated from the thigh bone muscle of a 2-month-old $\mathrm{C} 3 \mathrm{H}$ female mouse after crush injury [23]. This cell line does not differentiate when cultured in the medium with $10 \%-20 \%$ fetal bovine serum, and it maintains the stem cell characteristics of the satellite cell, with a rapid proliferation rate. Therefore, $\mathrm{C} 2 \mathrm{C} 12$ was selected as the skeletal muscle cell model in the present study. We found that $\mathrm{C} 2 \mathrm{C} 12$ proliferation and growth were inhibited differently with different concentrations of LY294002 and treatment times to inhibit the activity of PI3K. With the increased drug concentration and treatment, the inhibitory role was increasingly apparent, prompting us to explore the phenomenon.

4.1. PI3K-Dependent ILK Activation. PI3K plays an important role in the proliferation, growth, differentiation, and apoptosis of skeletal muscle cells $[12,24]$. The substrate of PI3K, phosphatidylinositol $(3,4,5)$-trisphosphate (PIP3) activates ILK and plays a key role in the regulation of the
$\mathrm{PKB} / \mathrm{AKT}$ and GSK-3 signaling pathways $[25,26]$. Therefore, in the present study the ILK activity was detected by inhibiting the activity of PI3K using PI3K-specific inhibitor LY294002. We found that ILK phosphorylation was significantly decreased. The results (Figure 1) confirmed that ILK activation was PI3K-dependent, which was consistent with other reports $[6,27]$.

4.2. ILK Negatively Regulates MEF2C Phosphorylation and Transcription Activity. ILK contains three domains to support a variety of functions, including the $\mathrm{N}$-terminal domain composed of four ankyrin repeats and a $\mathrm{PH}$ domain and the $\mathrm{C}$ terminal kinase domain. The unique protein structure enables ILK to play an important role in the coordination of extracellular matrix (ECM) and the signal transduction of growth factor $[28,29]$. Evidence suggests that ILK may directly phosphorylate proteins associated with tumorigenesis by increasing cell proliferation, enhancing anchor independence of growth factors, and inhibition of apoptosis [14, 30,31 . Investigations also revealed that MEF2C phosphorylation level increased significantly after ILK inhibition and knocking down, and the MCK expression that plays a vital role in the proliferation and differentiation of muscle cells increased significantly, suggesting that MEF2C transcription increased significantly after ILK inhibition or knocking down. After inhibiting ILK phosphorylation activity and mRNA expression, the phosphorylation activity of MEF2C and its transcriptional activity were significantly increased, thus indicating that ILK negatively regulates MEF2C phosphorylation and transcriptional activity.

4.3. PI3K Positively Regulates MEF2C Phosphorylation and Transcription. MEF2C directly regulates gene transcription of myomesin. MEF2C loss in skeletal muscle results in improper sarcomere organization. These results reveal a key role for MEF2C in maintenance of sarcomere integrity and postnatal maturation of skeletal muscle [32]. In the early stage, MEF2C phosphorylation was inhibited after PI3K inhibition [6]. Concomitantly, the expression of MCK mRNA regulated by $\mathrm{MEF} 2 \mathrm{C}$ was significantly decreased after inhibition of PI3K activity, which was also consistent with the results of MCK protein detection after PI3K inhibition [33].

4.4. Inhibition of PI3K-Dependent Activation of ILK Did Not Directly and Negatively Regulate MEF2C Phosphorylation and Transcription. ILK is activated in PI3K-dependent manner and regulates the related proteins or genes in the classical signal transduction pathway. In the present study, the ILK phosphorylation was inhibited after the inhibition of PI3K activity, when both MEF2C phosphorylation and transcription exhibited significantly decreasing trends. In order to further determine the regulatory effect of ILK on $\mathrm{MEF} 2 \mathrm{C}$, both the phosphorylation and the transcriptional activity of MEF2C displayed significantly increasing trends after direct inhibition of ILK phosphorylation and mRNA expression. Two different experimental results explain similar inhibition of ILK phosphorylation activity. According to previous studies, ILK is directly related to MEF2C, and 


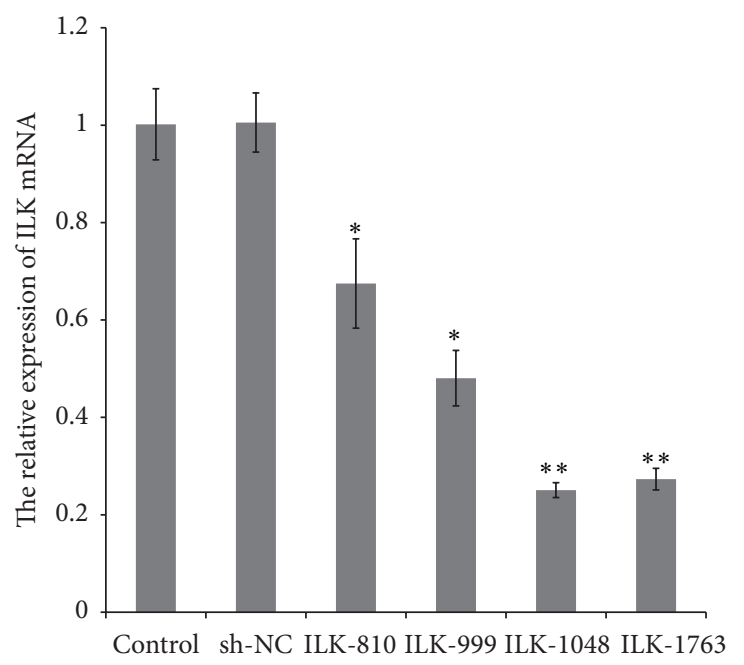

(a)

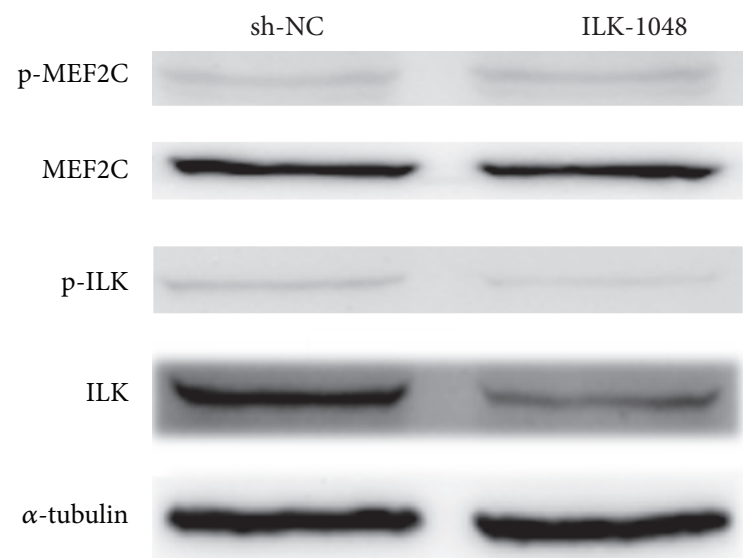

(c)

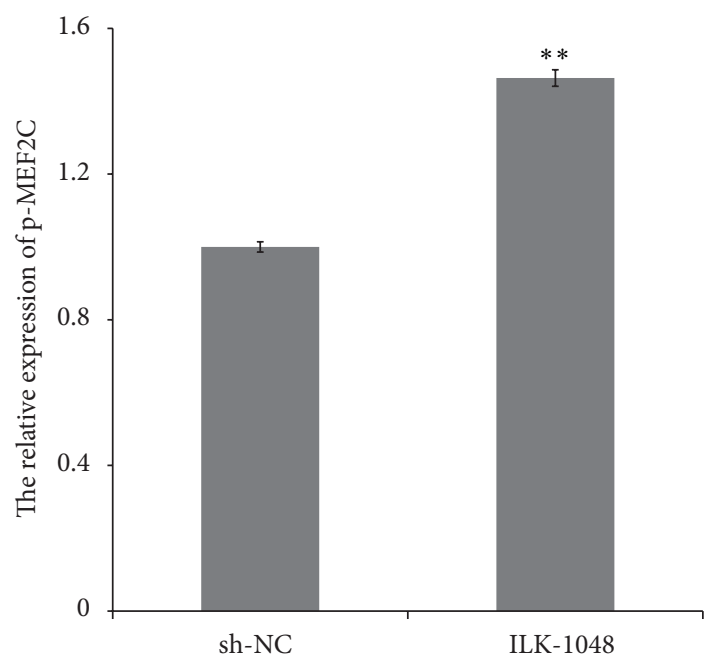

(e)
sh-NC ILK-810 ILK-999 ILK-1048 ILK-1763

ILK

$\alpha$-tubulin

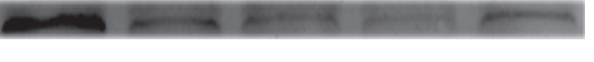

(b)

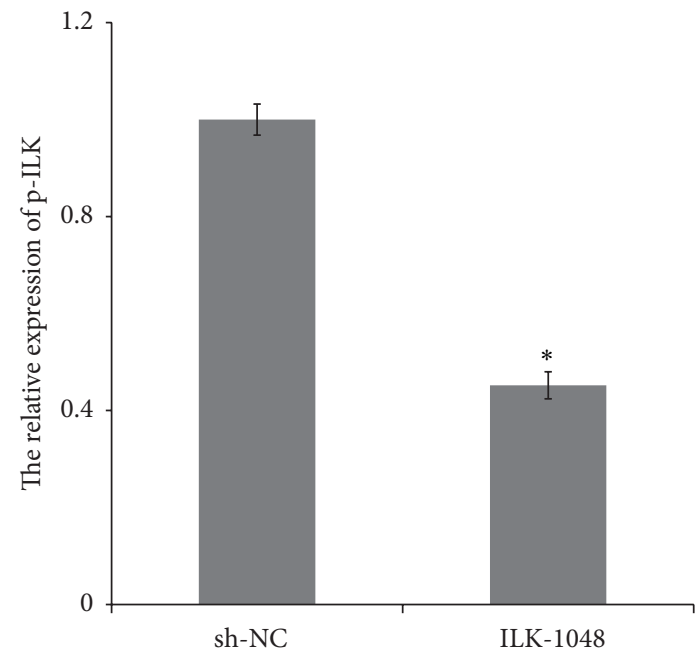

(d)

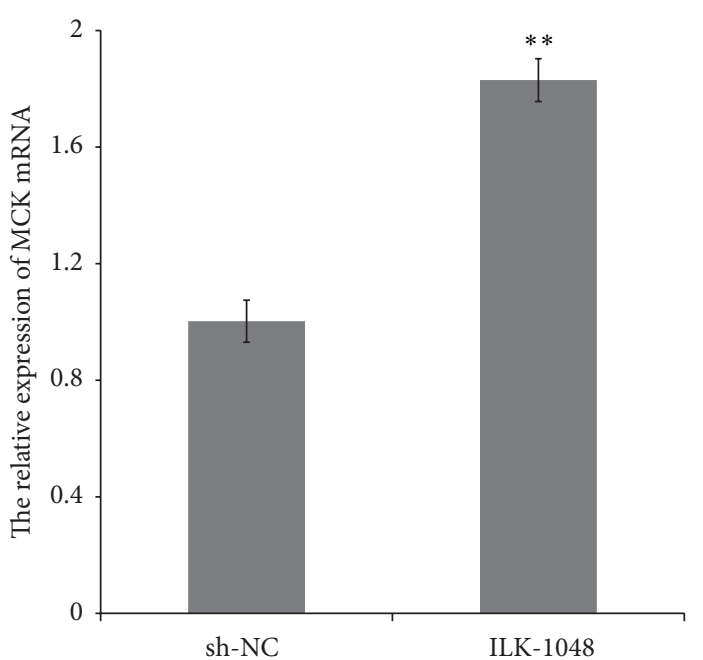

(f)

FIGURE 3: ILK-shRNA enhanced MEF2C phosphorylation activity and MCK mRNA expression. (a), (b) ILK-1048 was the most potent and selected for further study. (c), (d), and (e) Changes in MEF2C expression were not obvious with ILK-shRNA (ILK-1048) in C2C12 cells; ILK $(0.45204 \pm 0.027, p<0.05)$ phosphorylation levels decreased significantly and MEF2C phosphorylation levels increased significantly $(1.463772 \pm 0.022, p<0.01)$. (d) MCK mRNA levels in C2C12 cells treated with Cpd22 were significantly increased (1.829917 \pm 0.070 , $p<0.01)$ compared with that in the control. 


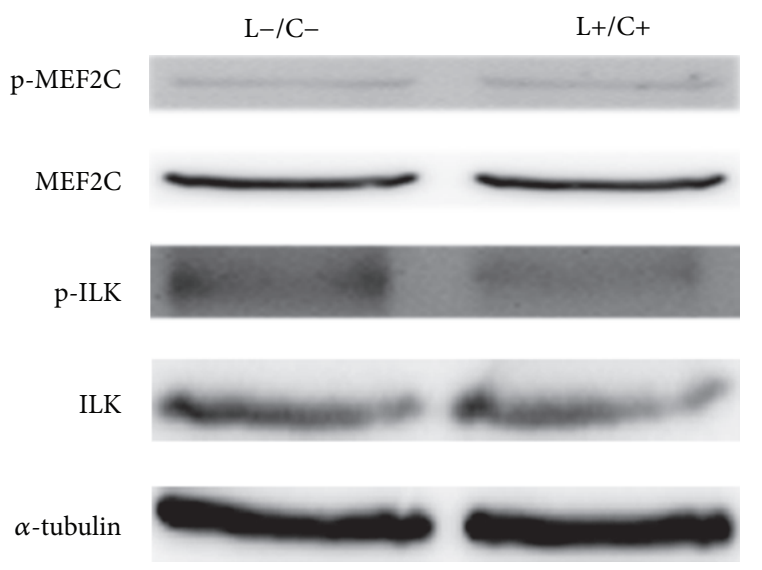

(a)

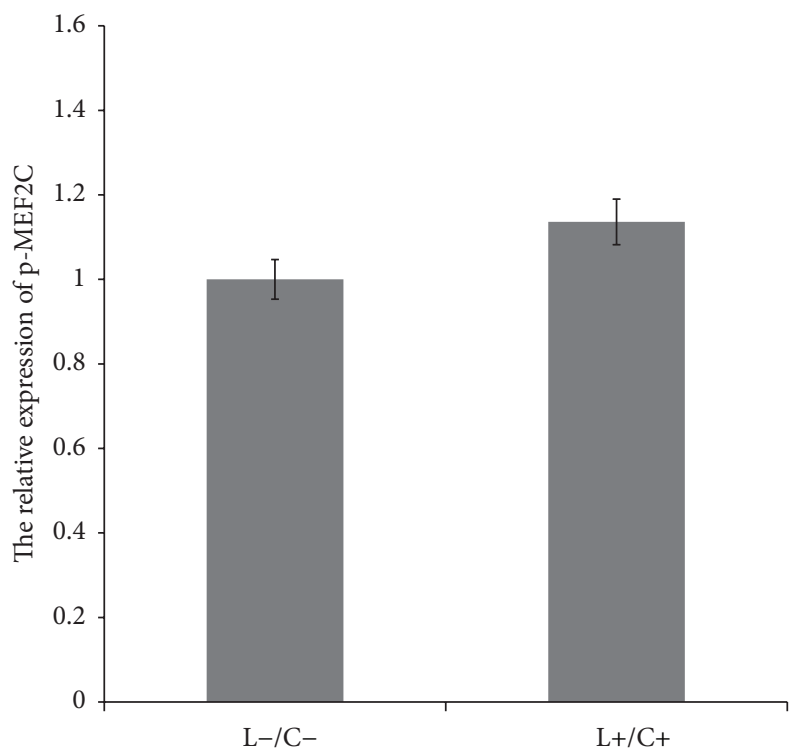

(c)

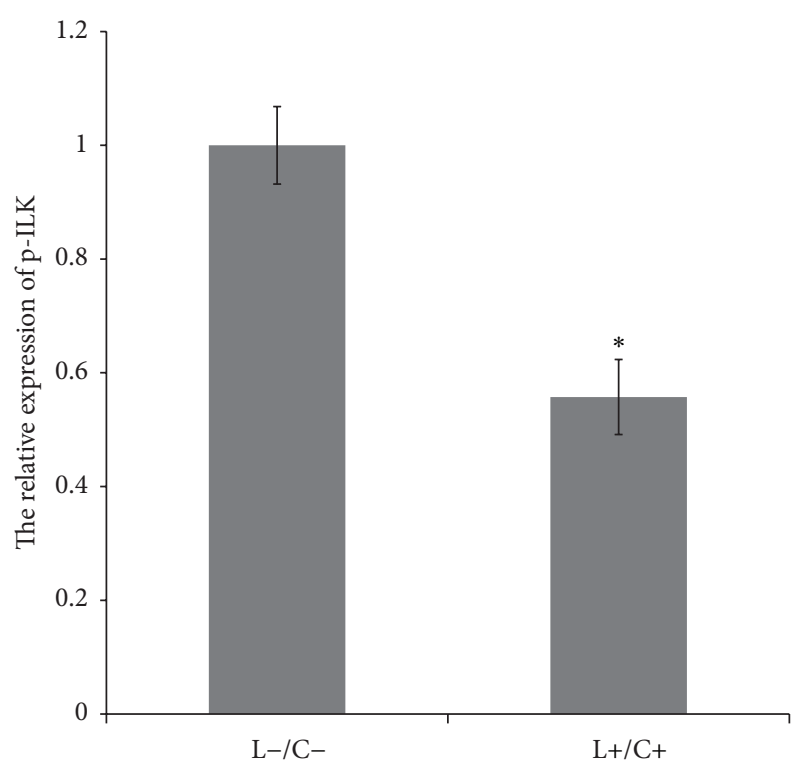

(b)

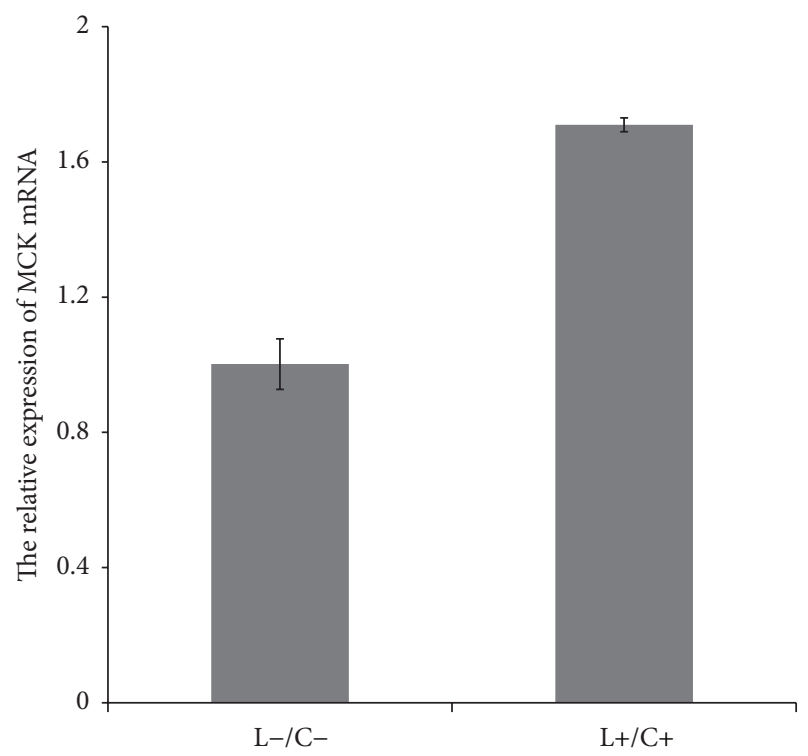

(d)

FIGURE 4: Inhibition of PI3K-dependent activation of ILK did not directly and negatively regulate MEF2C phosphorylation activity and MCK mRNA expression. (a), (b), and (c) Changes in ILK and MEF2C expression in LY294002/Cpd22-treated (L+/C+) C2C12 cells were not obvious; ILK $(0.557457 \pm 0.066, p<0.05)$ phosphorylation levels decreased significantly and MEF2C phosphorylation levels increased slightly $(1.1359755 \pm 0.054, p>0.05)$. (d) MCK mRNA levels in C2C12 cells treated with LY294002/Cpd22 were increased (1.7096923 \pm 0.020 , $p>0.05)$ compared with that in the control.

ILK negatively regulates the MEF2C phosphorylation and transcription activity. When two kinds of inhibitors were used, both the phosphorylation and transcription activities of MEF2C were increased, but lower than in the group treated with a single ILK inhibitor, which suggests that although the addition of PI3K inhibitor inhibited ILK activity, it did not negatively modulate MEF2C. Thus, we conclude that ILK activation independent of PI3K negatively regulates MEF2C phosphorylation and transcription in $\mathrm{C} 2 \mathrm{C} 12$ cells.

\section{Conclusions}

As one of the key molecules linking important signaling pathways inside and outside cells, ILK plays a stellar role in the study of skeletal muscle proliferation and tumor therapy. In this study, the relationship of the PI3K/ILK/MEF2C/MCK signaling pathway with muscle proliferation was investigated using $\mathrm{C} 2 \mathrm{C} 12$ cell line. A key finding: ILK activation was not entirely PI3K-dependent, especially in the regulation of 
MEF2C phosphorylation and transcription. Our next study will investigate how ILK regulates MEF2C in detail. We hope that the results of this study will provide a new impetus for the treatment of tumors and improvement of meat quality in domestic animals.

$\begin{array}{ll}\text { Abbreviations } \\ \text { DMEM: } & \text { Dulbecco's Modified Eagle's Medium } \\ \text { ECM: } & \text { Extracellular matrix } \\ \text { GSK3: } & \text { Glycogen synthase kinase } 3 \\ \text { ILK: } & \text { Integrin-linked kinase } \\ \text { LY294002: } & \text { 2-(4-Morpholino)-8-phenyl-4H-1- } \\ & \text { benzopyran-4-one } \\ \text { MCK: } & \text { Muscle-specific creatine kinase } \\ \text { MEF2: } & \text { Myocyte enhancer factor 2 } \\ \text { PI3K: } & \text { Phosphatidylinositol 3-kinase } \\ \text { PKB: } & \text { Protein kinase B. }\end{array}$

\section{Conflict of Interests}

The authors declare that there is no conflict of interests regarding the publication of this paper.

\section{Authors' Contribution}

Zhenguo Dong, Wei Pan, and Haiqing Wu contributed equally to this work.

\section{Acknowledgments}

Special thanks go to Haiqing Wu, Nimantana He, and Ming Cang for their help with the assistance and during the research. The funding of the research was from the National Science and Technology Major Projects (2014ZX08008-003).

\section{References}

[1] M. P. Thomas, J. Mills, and A.-M. Engelbrecht, "Phosphatidylinositol-3-kinase (PI3K) activity decreases in $\mathrm{C} 2 \mathrm{C} 12$ myotubes during acute simulated ischemia at a cost to their survival," Life Sciences, vol. 91, no. 1-2, pp. 44-53, 2012.

[2] G. Song, G. Ouyang, and S. Bao, "The activation of Akt/PKB signaling pathway and cell survival," Journal of Cellular and Molecular Medicine, vol. 9, no. 1, pp. 59-71, 2005.

[3] S. Brader and S. A. Eccles, "Phosphoinositide 3-kinase signalling pathways in tumor progression, invasion and angiogenesis," Tumori, vol. 90, no. 1, pp. 2-8, 2004.

[4] S. Sumitani, K. Goya, J. R. Testa, H. Kouhara, and S. Kasayama, "Aktl and Akt2 differently regulate muscle creatine kinase and myogenin gene transcription in insulin-induced differentiation of C2C12 myoblasts," Endocrinology, vol. 143, no. 3, pp. 820-828, 2002.

[5] B.-H. Jiang, J. Z. Zheng, and P. K. Vogt, "An essential role of phosphatidylinositol 3-kinase in myogenic differentiation," Proceedings of the National Academy of Sciences of the United States of America, vol. 95, no. 24, pp. 14179-14183, 1998.

[6] Y. Tamir and E. Bengal, "Phosphoinositide 3-kinase induces the transcriptional activity of MEF2 proteins during muscle differentiation," Journal of Biological Chemistry, vol. 275, no. 44, pp. 34424-34432, 2000.

[7] M. Delcommenne, C. Tan, V. Gray, L. Rue, J. Woodgett, and S. Dedhar, "Phosphoinositide-3-OH kinase-dependent regulation of glycogen synthase kinase 3 and protein kinase B/AKT by the integrin-linked kinase," Proceedings of the National Academy of Sciences of the United States of America, vol. 95, no. 19, pp. 1121111216, 1998.

[8] S. Dedhar, B. Williams, and G. Hannigan, "Integrin-linked kinase (ILK): a regulator of integrin and growth-factor signalling," Trends in Cell Biology, vol. 9, no. 8, pp. 319-323, 1999.

[9] A. Traister, S. Aafaqi, S. Masse et al., "ILK induces cardiomyogenesis in the human heart," PLoS ONE, vol. 7, no. 5, Article ID e37802, 2012.

[10] G. Hannigan, A. A. Troussard, and S. Dedhar, "Integrin-linked kinase: a cancer therapeutic target unique among its ILK," Nature Reviews Cancer, vol. 5, no. 1, pp. 51-63, 2005.

[11] J. Qin and C. Wu, "ILK: a pseudokinase in the center stage of cell-matrix adhesion and signaling," Current Opinion in Cell Biology, vol. 24, no. 5, pp. 607-613, 2012.

[12] S. Ghatak, J. Morgner, and S. A. Wickström, "ILK: a pseudokinase with a unique function in the integrin-actin linkage," Biochemical Society Transactions, vol. 41, no. 4, pp. 995-1001, 2013.

[13] P. C. McDonald, A. B. Fielding, and S. Dedhar, "Integrin-linked kinase-essential roles in physiology and cancer biology," Journal of Cell Science, vol. 121, no. 19, pp. 3121-3132, 2008.

[14] H. Wu, Y. Ren, W. Pan, Z. Dong, M. Cang, and D. Liu, “The mammalian target of rapamycin signaling pathway regulates myocyte enhancer factor-2C phosphorylation levels through integrin-linked kinase in goat skeletal muscle satellite cells," Cell Biology International, vol. 39, no. 11, pp. 1264-1273, 2015.

[15] M. J. Potthoff and E. N. Olson, "MEF2: a central regulator of diverse developmental programs," Development, vol. 134, no. 23, pp. 4131-4140, 2007.

[16] J. Xu, N. L. Gong, I. Bodi, B. J. Aronow, P. H. Backx, and J. D. Molkentin, "Myocyte enhancer factors $2 \mathrm{~A}$ and $2 \mathrm{C}$ induce dilated cardiomyopathy in transgenic mice," The Journal of Biological Chemistry, vol. 281, no. 14, pp. 9152-9162, 2006.

[17] R. J. van Oort, E. van Rooij, M. Bourajjaj et al., "MEF2 activates a genetic program promoting chamber dilation and contractile dysfunction in calcineurin-induced heart failure," Circulation, vol. 114, no. 4, pp. 298-308, 2006.

[18] J. D. Molkentin, L. Li, and E. N. Olson, "Phosphorylation of the MADS-box transcription factor MEF2C enhances its DNA binding activity," Journal of Biological Chemistry, vol. 271, no. 29, pp. 17199-17204, 1996.

[19] B. L. Black and E. N. Olson, "Transcriptional control of muscle development by myocyte enhancer factor-2 (MEF2) proteins," Annual Review of Cell and Developmental Biology, vol. 14, pp. 167-196, 1998.

[20] C. J. Vlahos, W. F. Matter, K. Y. Hui, and R. F. Brown, “A specific inhibitor of phosphatidylinositol 3-kinase, 2-(4-morpholinyl)8-phenyl-4H-1-benzopyran-4-one (LY294002)," Journal of Biological Chemistry, vol. 269, no. 7, pp. 5241-5248, 1994.

[21] K. Kimura, X. W. Cheng, A. Inoue, L. Hu, T. Koike, and M. Kuzuya, " $\beta$-Hydroxy- $\beta$-methylbutyrate facilitates PI3K/Aktdependent mammalian target of rapamycin and FoxO1/3a phosphorylations and alleviates tumor necrosis factor $\alpha$ /interferon $\gamma$-induced MuRF-1 expression in C2C12 cells," Nutrition Research, vol. 34, no. 4, pp. 368-374, 2014. 
[22] S.-L. Lee, E.-C. Hsu, C.-C. Chou et al., "Identification and characterization of a novel integrin-linked kinase inhibitor," Journal of Medicinal Chemistry, vol. 54, no. 18, pp. 6364-6374, 2011.

[23] D. Yaffe and O. Saxel, "Serial passaging and differentiation of myogenic cells isolated from dystrophic mouse muscle," Nature, vol. 270, no. 5639, pp. 725-727, 1977.

[24] C. Pasquali, D. Bertschy-Meier, C. Chabert et al., "A chemical proteomics approach to phosphatidylinositol 3-kinase signaling in macrophages," Molecular \& Cellular Proteomics, vol. 6, no. 11, pp. 1829-1841, 2007.

[25] Y. Qian, X. Zhong, D. C. Flynn et al., "ILK mediates actin filament rearrangements and cell migration and invasion through PI3K/Akt/Racl signaling," Oncogene, vol. 24, no. 19, pp. 31543165, 2005.

[26] W. J. Lee, "Insulin-like growth factor-I-induced androgen receptor activation is mediated by the $\mathrm{PI} 3 \mathrm{~K} /$ Akt pathway in C2C12 skeletal muscle cells," Molecules and Cells, vol. 28, no. 5, pp. 495-499, 2009.

[27] I. Serrano, M. L. Díez-Marqués, M. Rodríguez-Puyol et al., "Integrin-linked kinase (ILK) modulates wound healing through regulation of hepatocyte growth factor (HGF)," Experimental Cell Research, vol. 318, no. 19, pp. 2470-2481, 2012.

[28] M. Griera, E. Martin-Villar, I. Banon-Rodríguez et al., "Integrin linked kinase (ILK) regulates podosome maturation and stability in dendritic cells," The International Journal of Biochemistry \& Cell Biology, vol. 50, no. 1, pp. 47-54, 2014.

[29] M. D. F. B. Engelman, R. M. Grande, M. A. Naves, M. F. de Franco, and V. de Paulo Castro Teixeira, "Integrin-linked kinase (ILK) expression correlates with tumor severity in clear cell renal carcinoma," Pathology \& Oncology Research, vol. 19, no. 1, pp. 27-33, 2013.

[30] G. E. Hannigan, J. G. Coles, and S. Dedhar, "Integrin-linked kinase at the heart of cardiac contractility, repair, and disease," Circulation Research, vol. 100, no. 10, pp. 1408-1414, 2007.

[31] A. U. Ahmed, S. T. Sarvestani, M. P. Gantier, B. R. G. Williams, and G. E. Hannigan, "Integrin-linked kinase modulates lipopolysaccharide- and Helicobacter pylori-induced nuclear factor $\kappa \mathrm{B}$-activated tumor necrosis factor- $\alpha$ production via regulation of p65 serine 536 phosphorylation," The Journal of Biological Chemistry, vol. 289, no. 40, pp. 27776-27793, 2014.

[32] M. J. Potthoff, M. A. Arnold, J. McAnally, J. A. Richardson, R. Bassel-Duby, and E. N. Olson, "Regulation of skeletal muscle sarcomere integrity and postnatal muscle function by Mef2c," Molecular and Cellular Biology, vol. 27, no. 23, pp. 8143-8151, 2007.

[33] J. Li, Y. Zhang, N. Li et al., "Expression of Myogenin and MCK genes regulated by PI3K/AKT pathway," Yi Chuan, vol. 35, no. 5, pp. 637-642, 2013. 

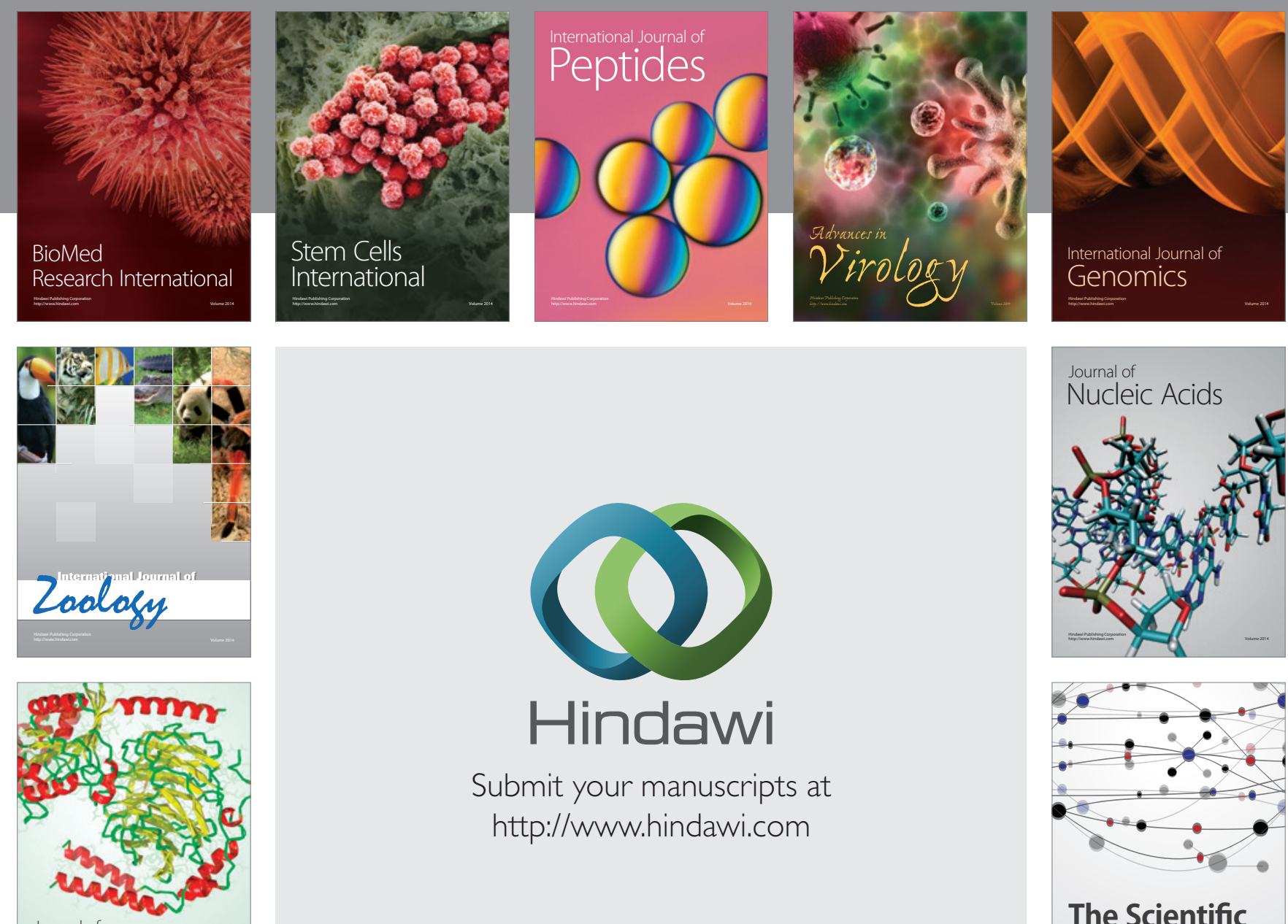

Submit your manuscripts at

http://www.hindawi.com

Journal of
Signal Transduction
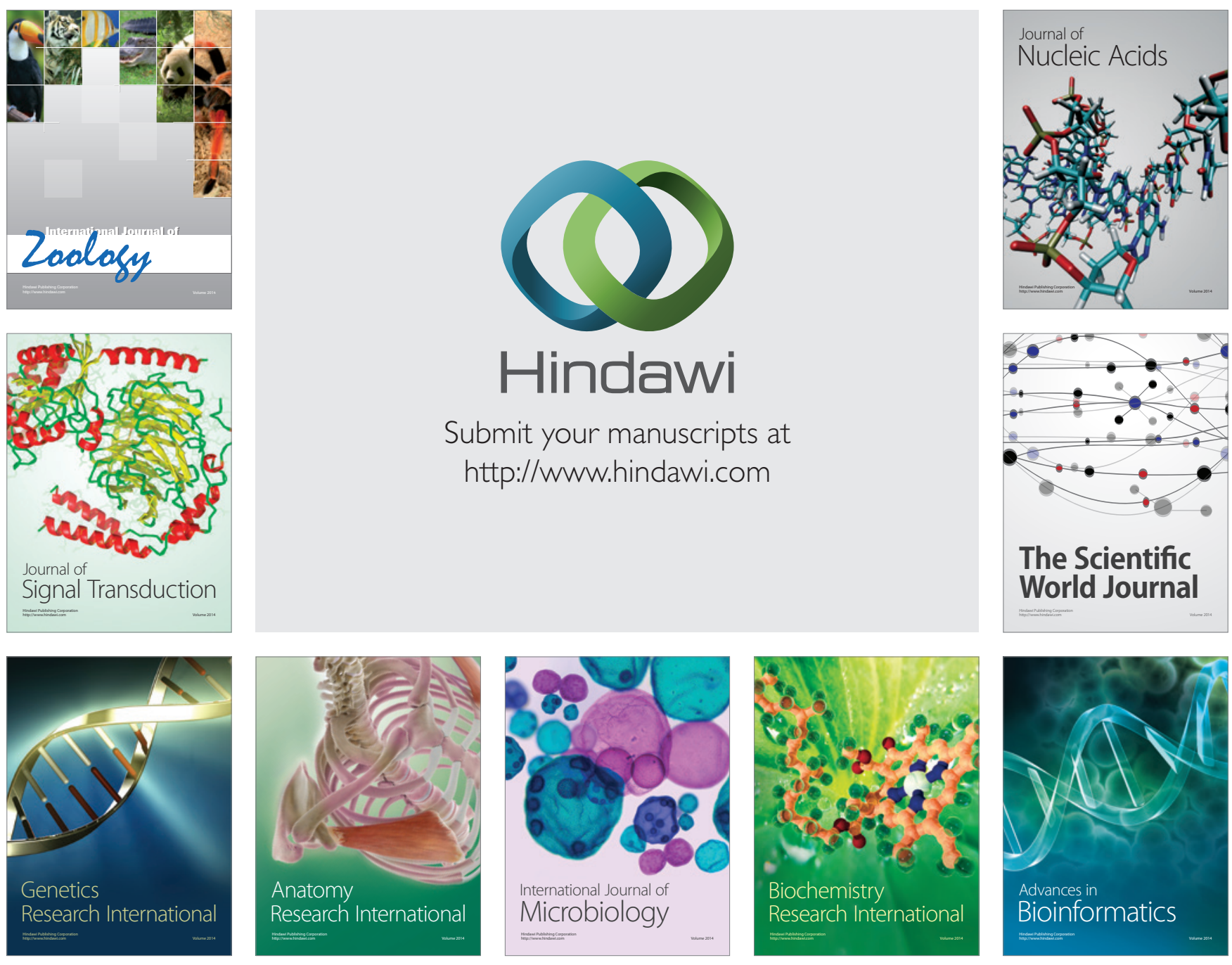

The Scientific World Journal
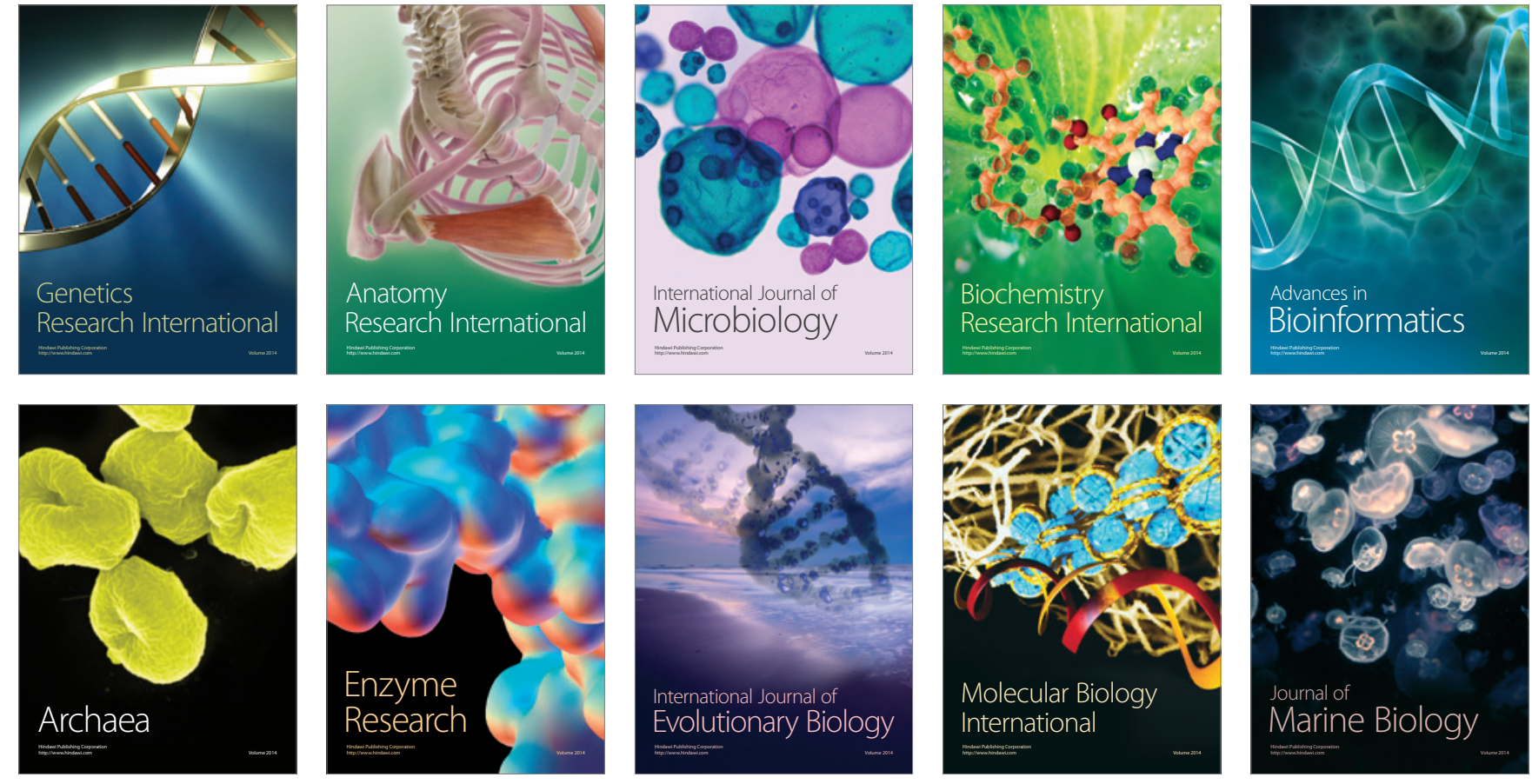